\title{
Tricoupled hybrid lattice Boltzmann model for nonisothermal drying of colloidal suspensions in micropore structures
}

\author{
Feifei Qin, ${ }^{1,2, *}$ Ali Mazloomi Moqaddam, ${ }^{2}$ Luca Del Carro, ${ }^{3}$ Qinjun Kang, ${ }^{4}$ Thomas Brunschwiler, ${ }^{3}$ \\ Dominique Derome, ${ }^{2}$ and Jan Carmeliet ${ }^{1}$ \\ ${ }^{1}$ Chair of Building Physics, Department of Mechanical and Process Engineering, ETH Zürich (Swiss Federal Institute of Technology in \\ Zürich), Zürich 8093, Switzerland \\ ${ }^{2}$ Laboratory of Multiscale Studies in Building Physics, Empa (Swiss Federal Laboratories for Materials Science and Technology), \\ Dübendorf 8600, Switzerland \\ ${ }^{3}$ Smart System Integration, IBM Research-Zürich, Saumerstrasse 4, 8803 Rüschlikon, Switzerland \\ ${ }^{4}$ Earth and Environment Sciences Division (EES-16), Los Alamos National Laboratory (LANL), Los Alamos, New Mexico 87545, USA
}

(Received 29 January 2019; published 23 May 2019)

\begin{abstract}
A tricoupled hybrid lattice Boltzmann model (LBM) is developed to simulate colloidal liquid evaporation and colloidal particle deposition during the nonisothermal drying of colloidal suspensions in micropore structures. An entropic multiple-relaxation-time multirange pseudopotential two-phase LBM for isothermal interfacial flow is first coupled to an extended temperature equation for simulating nonisothermal liquid drying. Then the coupled model is further coupled with a modified convection diffusion equation to consider the nonisothermal drying of colloidal suspensions. Two drying examples are considered. First, drying of colloidal suspensions in a two-pillar micropore structure is simulated in two dimensions (2D), and the final configuration of colloidal particles is compared with the experimental one. Good agreement is observed. Second, at the temperature of $343.15 \mathrm{~K}$ $\left(70^{\circ} \mathrm{C}\right)$, drying of colloidal suspensions in a complex spiral-shaped micropore structure containing 220 pillars is simulated (also in 2D). The drying pattern follows the designed spiral shape due to capillary pumping, i.e., transport of the liquid from larger pores to smaller ones by capillary pressure difference. Since the colloidal particles are passively carried with liquid, they accumulate at the small menisci as drying proceeds. As liquid evaporates at the small menisci, colloidal particles are deposited, eventually forming solid structures between the pillars (primarily), and at the base of the pillars (secondarily). As a result, the particle deposition conforms to the spiral route. Qualitatively, the simulated liquid and particle configurations agree well with the experimental ones during the entire drying process. Quantitatively, the model demonstrates that the evaporation rate and the particle accumulation rate slowly decrease during drying, similar to what is seen in the experimental results, which is due to the reduction of the liquid-vapor interfacial area. In conclusion, the hybrid model shows the capability and accuracy for simulating nonisothermal drying of colloidal suspensions in a complex micropore structure both qualitatively and quantitatively, as it includes all the required physics and captures all the complex features observed experimentally. Such a tricoupled LBM has a high potential to become an efficient numerical tool for further investigation of real and complex engineering problems incorporating drying of colloidal suspensions in porous media.
\end{abstract}

DOI: 10.1103/PhysRevE.99.053306

\section{INTRODUCTION}

Drying of colloidal suspensions is an interesting phenomenon observable in everyday life, for example, in the "coffee ring" [1,2] formed after drying of a coffee drop, and utilized commonly in industry, as with ink-jet printing [3]. This phenomenon has sparked the attention of researchers for a variety of applications such as the design of unique types of computer chips with high heat removal capacity [4-6] and innovative functional materials with better optical, magnetic, and electronic properties $[7,8]$. The accurate modeling of the drying of colloidal suspensions, a complex process involving coupled heat and mass transport and phase change, is very challenging.

\footnotetext{
*fqin@ethz.ch
}

In the past three decades, lattice Boltzmann modeling, which originated from the lattice gas automata method [9], has been developed into an efficient numerical approach to simulate and study different complex fluid flow problems ranging from turbulent $[10,11]$ and multiphase flows [12-16] to thermal $[17,18]$ and particulate flows $[19,20]$. For multiphase flows, there are in general four main categories of lattice Boltzmann models (LBMs): the Shan-Chen pseudopotential model [21,22], the free energy model [13,23,24], the colorgradient model [25], and the phase-field model [26,27]. Due to its simplicity and versatility, we apply the pseudopotential model which presents the intermolecular interactions with a density-dependent pseudopotential. The phase separation in this model is achieved by imposing a neighbor attraction between different phases; thus no interface tracking or reconstruction is needed. The original pseudopotential model suffers from the numerical instability when applied to large 
density ratio or high Reynolds or Weber number problems. By using (multirelaxation-time) entropic LBMs [12,13,16,23,24], the numerical stability is highly improved for simulating complex multiphase flows.

To simulate the thermal aspects of multiphase flow as displayed in evaporation, a temperature or energy equation considering latent heat has to be coupled to the aforementioned multiphase LBMs. There are three ways to implement this. The first method is to use multispeed LBM with more discrete velocities to recover higher-order moments than standard LBM does, such as the models presented in [28-31]. The benefit of this method is that only one set of distribution functions is needed, while the disadvantage is that the boundary condition for multispeed lattices becomes more difficult to apply, especially for problems with complex geometry such as porous media. The second way is to simply use the standard LBM population, but this approach will only recover the temperature equation correctly by taking care of the high-order moments, such as the models described in [32-34]. We mention that, for these two categories of thermal models, they are mainly used for single phase (compressible) flow in conjugation with heat transport, with small density difference. Another way is to solve the temperature or energy equation with the standard LBM plus a source term $[18,35-37]$ or to use a traditional numerical scheme like the finite difference method (FDM) [38,39]. When using LBM to recover the temperature equation, error terms always appear in the recovered macroscopic equation [40,41], which affect the accuracy of the model. In recent improvements [37,42], the errors have been reduced in such models and the results are close to that by FDM. Despite that, there is a numerical stability issue for very low diffusion coefficients [43,44]. However, the simulations using FDM have shown good results and high robustness in modeling boiling and evaporation $[38,39]$. Therefore, as we also deal with evaporation in this study, we use the FDM approach to solve the temperature equation.

Furthermore, to simulate the drying of a colloidal suspension, we also need to model particle transport and to couple this transport with the thermal multiphase model aforementioned. Basically, there are two categories of numerical models describing the transport of particles, i.e., Lagrangian models $[20,45,46]$ and Eulerian models [47-49]. In Lagrangian models, the movement of each individual particle is described with Newton's second law. Particle motions in binary immiscible fluids [50] and isothermal drying of a colloidal drop [51] have been previously simulated with the Lagrangian method. Similarly in [52], massless tracers are used to model particle motions inside the liquid droplet and around the liquid-vapor interface during droplet drying. Although the Lagrangian models can provide more accurate results, the computational costs become prohibitively high when a huge number of particles are involved [53]. In Eulerian models, particles are represented as solute concentration described with a convection diffusion equation. In this way, Eulerian models can deal with a large number of particles. Eulerian models have already shown their capabilities in studying solute transport [54], crystallization [55,56], and efflorescence [57]. For the colloidal suspension under investigation here, we adopt an Eulerian scheme because of its computational efficiency as over millions of particles are present. In this study, we propose a modified convection diffusion equation (MCDE) that considers the fluid-particle interaction to model particle transport, accumulation at the vapor-liquid interface, and deposition, defined here as the process forming a growing nonporous solid. Similar to the extended temperature equation (ETE), we use the FDM approach to solve the MCDE. Coupling the MCDE to the thermal multiphase model enables us to simulate and study the nonisothermal drying of colloidal suspensions both qualitatively and quantitatively. Since the two-phase flow is solved by LBM while the ETE and MCDE are solved by FDM, the proposed model is referred to as a tricoupled hybrid lattice Boltzmann model. This model was previously applied to study the self-assembly of clogging structures by evaporation of colloidal suspensions in twodimensional (2D) porous media [58], analyzing the effects of initial concentration and porosity on the formation process of self-assembly. However, in [58], we mainly focused on the physics of particle deposition and the correspondingly created clogging structures without details in the numerical implementations and on model developments. In this paper, we aim to describe the model in detail regarding how different submodels are coupled and the boundary conditions implemented.

The remainder of this paper is structured as follows: We firstly describe the three submodels, namely, the isothermal two-phase flow, the temperature equation, and the particle transport and deposition process, in Sec. II. Then we validate our model simulating the drying of colloidal suspensions in a simple two-pillar system in Sec. III A. We then continue with Sec. III B where the drying of colloidal suspensions in a spiral-shaped pillar system is studied and compared with experimental results. Finally, in Sec. IV, we evaluate the proposed model and summarize the paper. We mention that all the simulations in our study here are in 2D.

\section{NUMERICAL MODEL}

\section{A. Isothermal two-phase flow}

We already showed in former work that the entropic multiple-relaxation-time multiphase lattice Boltzmann model (EMRT-MP LBM) [12] performs well in simulating the complex isothermal interfacial flow of droplet dynamics. Here we use this approach to model the evolution of the liquid-vapor interface during drying of a colloidal solution in a porous medium. The original entropic multiple-relaxation time for single phase flow was proposed in [59] and analyzed in detail in [10]. For multiphase flow, the LB equation for the populations $f_{i}(\mathbf{x}, t)$ of discrete velocities $\mathbf{v}_{i}, i=1, \ldots, Q$ that incorporates external force is written as

$$
\begin{aligned}
f_{i}\left(\mathbf{x}+\mathbf{v}_{i}, t+1\right)= & f_{i}^{\prime} \equiv(1-\beta) f_{i}(\mathbf{x}, t)+\beta f_{i}^{\mathrm{mirr}}(\mathbf{x}, t) \\
& +f_{i}^{\mathrm{eq}}(\rho, \mathbf{u}+\Delta \mathbf{u})-f_{i}^{\mathrm{eq}}(\rho, \mathbf{u}) .
\end{aligned}
$$

The equilibrium populations $f_{i}^{\text {eq }}$ maximize the entropy $S[f]=-\sum_{i=1}^{Q} f_{i} \ln \left(f_{i} / W_{i}\right)$ under fixed constraints satisfying density and momentum conservations $\{\rho, \rho \mathbf{u}\}=$ $\sum_{i=1}^{Q}\left\{1, \mathbf{v}_{i}\right\} f_{i}^{\text {eq }}$, where $W_{i}$ are the lattice weights $[60,61]$. For 
D2Q9 lattices, the weights are $W_{0}=4 / 9, W_{1, \ldots, 4}=1 / 9$, and $W_{5, \ldots, 8}=1 / 36$. The parameter $0<\beta<1$ is determined by the kinematic viscosity $v$ through $v=c_{s}^{2}[1 /(2 \beta)-1 / 2] \delta t$. Here $c_{s}=\delta x /(\sqrt{3} \delta t)$ is the lattice speed of sound, and lattice units $\delta x=\delta t=1$ are used with lattice speed $c=1$. The mirror state $f_{i}^{\text {mirr }}$ is constructed at each lattice site and every time step from the entropy maximization of the summarized postcollision population $f_{i}^{\prime}$ by relaxing high-order moments properly $[10,59]$.

In Eq. (1), the last two terms $f_{i}^{\mathrm{eq}}(\rho, \mathbf{u}+\Delta \mathbf{u})-f_{i}^{\mathrm{eq}}(\rho, \mathbf{u})$ represent the fluid-fluid cohesive force for phase separation and the fluid-solid interaction for realizing various wettabilities. The forces are implemented by evaluation of the flow velocity increment $\Delta \mathbf{u}=\mathbf{F} \delta t / \rho$ with the total force $\mathbf{F}=$ $\mathbf{F}_{c}+\mathbf{F}_{w}$. To obtain a tunable surface tension, the multirange pseudopotential-based cohesive force is applied as [62]

$$
\mathbf{F}_{c}=-\psi(\mathbf{x}) \sum_{i=1}^{Q} w\left(\left|\mathbf{v}_{i}\right|^{2}\right)\left[G_{1} \psi\left(\mathbf{x}+\mathbf{v}_{i}\right)+G_{2} \psi\left(\mathbf{x}+2 \mathbf{v}_{i}\right)\right] \mathbf{v}_{i},
$$

where the interaction potential is presented as

$$
\psi=\sqrt{2\left(P_{\mathrm{EoS}}-\rho c_{s}^{2}\right) /\left[\left(G_{1}+2 G_{2}\right) c^{2}\right]},
$$

and $G_{1}, G_{2}$ are coefficients to tune the surface tension. In our simulations, $G_{1}$ and $G_{2}$ are set as $G_{1}=-1.0$ and $G_{2}=0$, yielding a surface tension of $\sigma=1.05 \times 10^{-2}$ in lattice units. For D2Q9 lattices, the weights are $w(1)=1 / 3$ and $w(2)=$ $1 / 12 . P_{\mathrm{EoS}}$ is the equation of state (EoS) and here we adopt the Carnahan-Starling (CS) EoS [63]:

$$
P_{\mathrm{EoS}}=\rho R T \frac{1+b \rho / 4+(b \rho / 4)^{2}-(b \rho / 4)^{3}}{(1-b \rho / 4)^{3}}-a \rho^{2},
$$

in which the attraction and repulsion parameters are $a=$ $0.4963 R^{2} T_{c}^{2} / p_{c}, b=0.18727 R T / p_{c} . T_{c}$ and $p_{c}$ represent the critical temperature and pressure, respectively. $T$ is the temperature and $R$ is the gas constant. In simulations, the parameters are set to $a=2 / 49, b=2 / 21$, and $R=1$.

The other force fluid-solid interaction $\mathbf{F}_{w}$ is implemented as [12]

$$
\begin{aligned}
\mathbf{F}_{w}= & -\psi(\mathbf{x}) \sum_{i=1}^{Q} w\left(\left|\mathbf{v}_{i}\right|^{2}\right)\left[G_{1} \psi\left(r_{w}\right) I\left(\mathbf{x}+\mathbf{v}_{i}\right)\right. \\
& \left.+G_{2} \psi\left(r_{w}\right) I\left(\mathbf{x}+2 \mathbf{v}_{i}\right)\right] \mathbf{v}_{i},
\end{aligned}
$$

where $I$ is the indicator function that equals unity at solid nodes and zero at fluid nodes, and $r_{w}$ the parameter to determine wettability. $w\left(\left|\mathbf{v}_{i}\right|^{2}\right)$ in $\mathbf{F}_{c}$ and $\mathbf{F}_{w}$ are appropriately chosen weights as in Eq. (2). Real velocity of the fluid including the force term is $\mathbf{u}_{f}=\mathbf{u}+\Delta \mathbf{u} / 2$.

With the proposed EMRT-MP LBM in [12], we have shown thermodynamic consistency by the coexistence curve of liquid and vapor density at different temperatures, high accuracy by reducing spurious currents around the liquidvapor interface by about one order of magnitude, and stability for interfacial flow at the high Reynolds number of 5000 .

\section{B. Extended temperature equation}

The heat transport equation is derived from the local balance law for entropy [64] by neglecting viscous heat dissipation:

$$
\rho T \frac{D s}{D t}=\nabla \cdot(\lambda \nabla T),
$$

where $s$ is the entropy, $\rho$ is the fluid density, $\lambda$ is the thermal conductivity, and $T$ is the temperature. $D(\cdots) / D t=$ $\partial t(\cdots)+\mathbf{u}_{f} \cdot \nabla(\cdots)$ is the material derivative with $\mathbf{u}_{f}$ representing local fluid velocity. With the thermodynamic relations of a nonideal gas, the following equation is obtained:

$$
D s=\frac{C_{V}}{T} D T+\left(\frac{\partial P_{\mathrm{EoS}}}{\partial T}\right)_{V} D V,
$$

where $C_{V}$ is the specific heat at constant volume and $V=1 / \rho$ is the specific volume. With continuum equation $D \rho / D t=$ $-\rho \boldsymbol{\nabla} \cdot \mathbf{u}_{f}$, the governing equation for temperature transport can be derived as $[39,65]$

$$
\begin{aligned}
\partial_{t} T= & -\mathbf{u}_{f} \cdot \nabla T+\frac{1}{\rho C_{V}} \nabla \cdot(\lambda \nabla T) \\
& -\frac{T}{\rho C_{V}}\left(\frac{\partial P_{\mathrm{EoS}}}{\partial T}\right)_{\rho} \nabla \cdot \mathbf{u}_{f} .
\end{aligned}
$$

The first two terms on the right-hand side of Eq. (8) represent heat convection and conduction, respectively, while the last term corresponds to the latent heat for phase change. This equation is solved with the finite difference method with a second-order Runge-Kutta scheme for time discretization:

$$
T^{t+\delta t}=T^{t}+\frac{\delta t}{2}\left(h_{1}+h_{2}\right),
$$

where $h_{1}, h_{2}$ are given as

$$
h_{1}=F\left(T^{t}\right), \quad h_{2}=F\left(T^{t}+\delta t h_{1}\right),
$$

and $F(T)$ represents the right-hand side of Eq. (8) while $\delta t$ is the time step in numerical time discretization. For spatial discretization, isotropic central schemes are employed to evaluate the first-order derivative and the Laplacian $[66,67]$.

To model nonisothermal evaporation, the extended temperature equation [ETE, Eq. (8)] is coupled to the EMRTMP LBM [Eq. (1)], referred to as T-EMRT-MP LBM. The two-way coupling works this way: At each time step, flow variables like density $\rho$ and velocity $\mathbf{u}_{f}$ are computed by solving Eq. (1), and pressure $P_{\text {Eos }}$ by Eq. (4); then they are plugged into Eq. (8) to update the temperature field $T$. Finally the updated temperature $T$ is used in computing the flow field with the equation of state $P_{\mathrm{EoS}}$ for the next time step. As the two-way coupling proceeds, the drying evolves. We mention that both equations are solved with the same mesh to avoid the need for interpolation.

With the coupling model, nonisothermal drying of liquid in various geometries under different temperatures is simulated and compared with experimental and theoretical data in [67], showing high accuracy and stability.

\section{Particle transport and deposition}

As discussed before, we apply the Eulerian model to simulate particle transport. By representing particle concentration 
and particle velocity as $\phi$ and $\mathbf{u}_{p}$, we write the MCDE as

$$
\frac{\partial \phi}{\partial t}+\nabla \cdot\left(\phi \mathbf{u}_{p}\right)=\nabla \cdot\left(D_{p} \nabla \phi\right)
$$

where the particle velocity $\mathbf{u}_{p}$ is modified from the fluid velocity $\mathbf{u}_{f}$ considering two-phase flow:

$$
\mathbf{u}_{p}=\mathbf{u}_{f, m}+\Delta \mathbf{u}_{p} .
$$

Here $\mathbf{u}_{f, m}=\mathbf{u}_{f}$ in liquid while $\mathbf{u}_{f, m}=0$ in vapor since particles are present only within the liquid phase. The term $\Delta \mathbf{u}_{p}=\mathbf{F}_{p} \delta t / \rho_{p}$ models the fluid-particle interaction $\mathbf{F}_{p}$ to let particles accumulate around the liquid-vapor interface instead of flowing into vapor during evaporation. $\rho_{p}$ is the particle density. The particle concentration $\phi=n_{p} V_{p} / V_{L}$ is the volume fraction of particle and lattice, where $n_{p}, V_{p}$, and $V_{L}$ are the number of particles, particle volume, and lattice volume, respectively. The fluid-particle interaction $\mathbf{F}_{p}$ is modeled by making an analogy to the fluid-solid interaction $\mathbf{F}_{w}$ in Eq. (5) while considering particle size and local concentration:

$$
\begin{aligned}
\mathbf{F}_{p}= & -\phi C_{s, p} \psi\left(\rho_{p}\right) \sum_{i=1}^{Q} w\left(\left|\mathbf{v}_{i}\right|^{2}\right)\left[G_{1} \psi^{\prime}\left(\mathbf{x}+\mathbf{v}_{i}\right)\right. \\
& \left.+G_{2} \psi^{\prime}\left(\mathbf{x}+2 \mathbf{v}_{i}\right)\right] \mathbf{v}_{i},
\end{aligned}
$$

where $C_{s, p}=S_{p} / S_{L}$ is the particle size coefficient, and $S_{p}, S_{L}$ are particle surface area and lattice surface area $\left(S_{L}=1\right.$ in 2D LBM), respectively. The particle size coefficient $C_{s, p}$ is the ratio of particle surface area to lattice surface area and reflects the influence of particle size. This is different from the concentration $\phi=n_{p} V_{p} / V_{L}$ which indicates the volume fraction of local particles per unit lattice volume $\left(V_{L}=1\right.$ in 2D LBM). For example, with the same $\phi$, smaller $C_{s, p}$ means the number of particles is higher. Thus, $\mathbf{F}_{p}$ represents the fluid-particle interaction of a certain number of particles with a specific size. The current use of the convection diffusion equation to model particle transport and deposition does not allow taking into account the fluid-particle interaction difference $\mathbf{F}_{p}$ induced by particle size. Thus, in this work, the particle size coefficient is set to be $C_{s, p}=1$. When the local volume concentration reaches $\phi=1$, the particle deposits to become solid. With the setting of $C_{s, p}=1$, the fluid-particle interaction $\mathbf{F}_{p}$ [Eq. (13)] degenerates to the form of fluid-solid interaction $\mathbf{F}_{w}$ [Eq. (15)]. This transition of forces is in accordance with the physical process of deposition. Upcoming development of the model about $C_{s, p}$ will allow different particle sizes to be considered.

In Eq. (11), $D_{p}$ is the diffusion coefficient derived using the Stokes-Einstein equation as $D_{p}=k_{B} T /\left(6 \pi \mu_{L} R_{p}\right)$, where $k_{B}$ is the Boltzmann constant; $R_{p}$ is the particle radius; $T$ and $\mu_{L}$ are the temperature and viscosity of the liquid, respectively [47]. In our experimental systems, the heating temperature is $70^{\circ} \mathrm{C}$ when the water viscosity is $\mu_{L}=4.04 \times 10^{-4} \mathrm{~N} \mathrm{~s} / \mathrm{m}^{2}$. The nanoparticles are polystyrene spheres of radius $R_{p}=$ $500 \mathrm{~nm}$. Then the calculated diffusion coefficient comes to $D_{p}=1.24 \times 10^{-12} \mathrm{~m}^{2} / \mathrm{s}$, which is very small. The Péclet number $\mathrm{Pe}_{p}=\bar{U} L_{0} / D_{p}$ is used to evaluate the dominant transport mechanism between convection and diffusion. In the experiment included in this paper, as shown in Fig. 7, the average velocity of the liquid front is $\bar{U}=1.54 \times 10^{-4} \mathrm{~m} / \mathrm{s}$

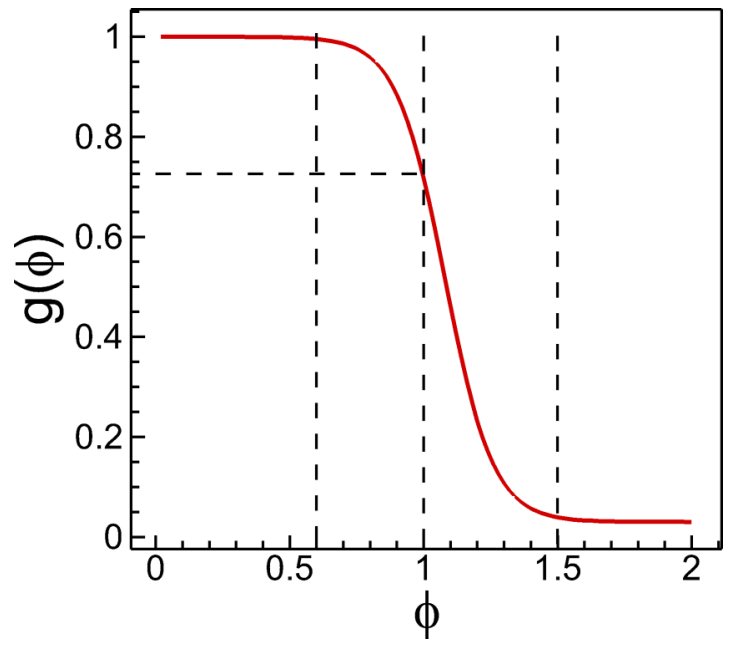

FIG. 1. The relation between local particle concentration $\phi$ and corresponding interaction potential $g(\phi)$.

and the characteristic length is $L_{0}=3.75 \times 10^{-4} \mathrm{~m}$ (shown in Fig. 5), leading to $\mathrm{Pe}_{p}(\exp )=4.64 \times 10^{4}$ revealing convection is dominant for particle transport. In our simulations, we aim for a similar Péclet number in order to secure the same physics. Using $D_{p}=3.5 \times 10^{-3}$ (lattice units), the liquid front velocity $\bar{U}=8.9 \times 10^{-3}$ (lattice units), and the characteristic length $L_{0}=60$ lattices, the Péclet number corresponding to the simulation is $\mathrm{Pe}_{p}(\mathrm{sim})=1.53 \times 10^{2}$. It is not as large as the one in the experiment, but it also indicates that convection is dominant.

We recall that, according to Eqs. (3) and (4), the interaction potential $\psi=\psi(\rho, T)$ is a function of density $\rho$ and temperature $T$. In Eq. (13), we actually use a modified potential $\psi^{\prime}=\psi^{\prime}[\rho g(\phi), T]$ in the summation which considers the local particle concentration $\phi$. Physically, with increasing $\phi$, the local volume fraction of the liquid is getting smaller, which reduces the interaction potential. We apply $g(\phi)$ here to mimic this effect in each lattice in our model. Our simulation results are in good agreement with those observed by experiments by considering a hypertangent function for $g(\phi)$ as follows:

$$
g(\phi)=\frac{\rho_{V}}{\rho_{L}}+\frac{1}{2}\left(1-\frac{\rho_{V}}{\rho_{L}}\right)\left\{1-\tanh \left[\left(\frac{\phi}{2}-A\right) / B\right]\right\} .
$$

The parameters $A$ and $B$ in the function determine the deposition of the particles. In this paper, we choose $A=0.54$ and $B=0.09$ which yields a particle deposition configuration agreeing with experimental results. The function $g(\phi)$ shown in Fig. 1 indicates that for local concentration $\phi<0.6$, the liquid behavior is not affected by the presence of particles. When the local concentration is higher than $0.6, g(\phi)$ decreases and then the potential difference between the local spot and its neighbors results in diffusion from higher to lower concentration. Due to diffusion, the local concentration becomes 1.0 only when the suspension evaporates to a certain small volume where all neighbor concentrations are 1.0. Under this condition, the suspensions in this volume deposit and become solid simultaneously. The bracket of values for $\phi$ is set as $(0.0,2.0)$, as shown in Fig. 1, to ensure numerical stability, 
since singularity concentration values may occasionally be a little higher than 1.0 in the simulations. The two requirements for solid structure deposition are the local concentration reaches a threshold of 1.0 and at least one of the neighbors is already solid or its concentration also reaches 1.0 [68], namely,

$$
\begin{aligned}
& \phi(\mathbf{x}) \geqslant 1.0, \\
& \exists i \in\{1,2, \ldots, 8\}, \text { such that } \phi\left(\mathbf{x}+\mathbf{v}_{i}\right) \geqslant 1.0|| I\left(\mathbf{x}+\mathbf{v}_{i}\right)=1 .
\end{aligned}
$$

In the proposed model, we do not consider the local effect of particle concentration on the liquid properties like viscosity, surface tension, and thermal conductivity, since this higher concentration may only play a role over a small area close to the liquid-vapor interface. The coupling between T-EMRTMP LBM and particle transport and deposition model is a oneway coupling; i.e., the particle velocity is modified from fluid velocity obtained from T-EMRT-MP LBM while the newly formed particle bridges do not affect the fluid flow. We explain this choice and its implications next. The coupling between TEMRT-MP LBM and particle transport and deposition model could also be implemented as two way, as the model has the capability to obtain the new flow fields based on the evolving microstructure due to particle deposition, as done previously in [58]. The reason we use one-way coupling here is that the initial concentration of the colloidal suspension is very low (2\%). With this low concentration, the formed particle bridges in experiment are hollow structures which have very small influences on fluid flow. Since the simulation is in $2 \mathrm{D}$, hollow structures along the height of the pillars in the vertical direction cannot be modeled. However, using one-way coupling allows us to model fluid, mainly vapor, flow through the then-assumed-to-be hollow structures (Fig. 8) as observed in the experiment. With the proposed coupling model, nonisothermal drying of colloidal suspensions can be simulated and studied physically.

Similar to the extended temperature equation, the MCDE is solved by the finite difference method with a secondorder Runge-Kutta scheme. Moreover, a zero flux boundary condition is enforced at the solid boundary, as discussed in Sec. III, to make sure that the total particle mass is conserved. The validation of the tricoupled model is shown in Sec. III A with the drying of a colloidal suspension in a two-micropillar system.

\section{RESULTS AND DISCUSSION}

\section{A. Two-micropillar system}

\section{Setups and boundary conditions}

To validate our tricoupled model here, we simulate the drying of a colloidal suspension in a very simple geometry with only two square micropillars separated by a distance equal to twice their side dimension. We use three different sizes of pillars $\left(8 \times 8,12 \times 12\right.$, and $16 \times 16$ lattices $\left.^{2}\right)$ to check the consistency of our model. The ratios of other dimensions to pillar size are maintained constant in the three simulations. The simulation domain and setup with the pillar size $16 \times$ 16 lattices $^{2}$ are illustrated in Fig. 2. The pillars are made of silicon with a contact angle with water varying between $35^{\circ}$ and $39^{\circ}$. Since the ratio of length or width to height is sufficiently high in the experiment [6], the micropillar system is considered as quasi-2D and therefore a $2 \mathrm{D}$ simulation is performed here. The simulated liquid to vapor density ratio is $\rho_{L} / \rho_{V} \approx 30\left(\rho_{L}=0.33, \rho_{V}=0.011\right)$ under a saturation temperature $T_{\text {sat }}=0.75 T_{c}$, where $T_{c}$ is the critical temperature. The kinematic viscosity of the liquid and vapor is set as $v_{L}=$ $v_{V}=0.167$. The thermal diffusivities of liquid and vapor are $\alpha_{L}=0.24$ and $\alpha_{V}=0.036$ in lattice units. The pillar surfaces are treated as nonslip walls. Initially, the pillar and colloidal suspension temperature is set to $T_{p}=0.76 T_{c}$ while vapor temperature is fixed at saturation temperature. The colloidal suspension evaporates because of pressure difference induced by the temperature difference between $T_{p}$ and $T_{\text {sat }}$. The pillar temperature remains at $T_{p}$ to continuously supply energy for evaporation. The temperature difference is $d T=T_{p}-T_{\text {sat }}=$ $0.01 T_{c}$, which is a quite low value. We mention that our model can deal with a tenfold larger temperature difference and remain stable [67]. The reason we use the small temperature difference is to ensure drying mechanisms similar to what occurred in the experiment, i.e., where the capillary force is much more dominant than the viscous force. Both the fluid velocity and temperature at the four lateral boundaries are set as zero gradient as $\partial \mathbf{u}_{f} / \partial \mathbf{n}=0, \partial T / \partial \mathbf{n}=0$, so that the evaporated vapor flows out of the domain freely.

The initial concentration in the $3 \mathrm{D}$ experiment is $2.0 \%$ while, in the $2 \mathrm{D}$ simulation, we set $\phi_{0}=0.12$ in the colloidal suspension. The reason for the difference is that, in the $3 \mathrm{D}$ experiment, the solid formed by the nanoparticles is not uniform over height, but more like a hollow structure. For our 2D simulations, we try to compare the top view with experiment, which results in the particle deposition being uniform over height. Thus, the initial particle concentration in 2D

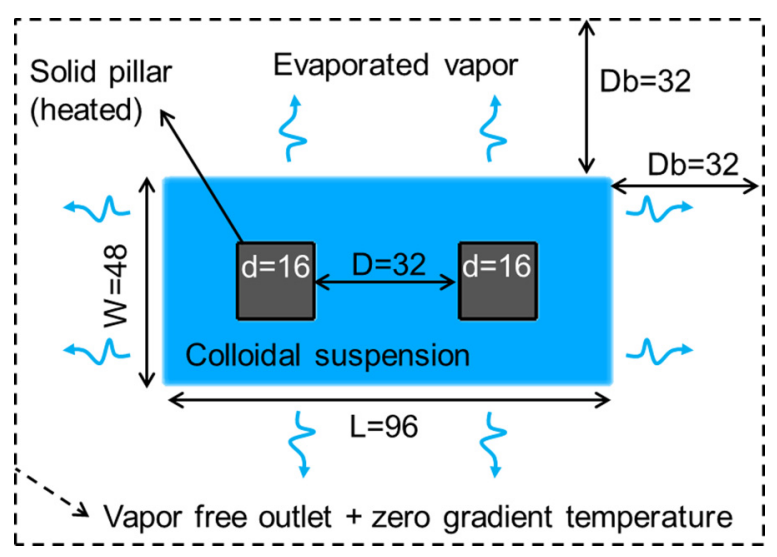

FIG. 2. Simulation domain and setup of the two-pillar system (all dimensions are in lattice units). 


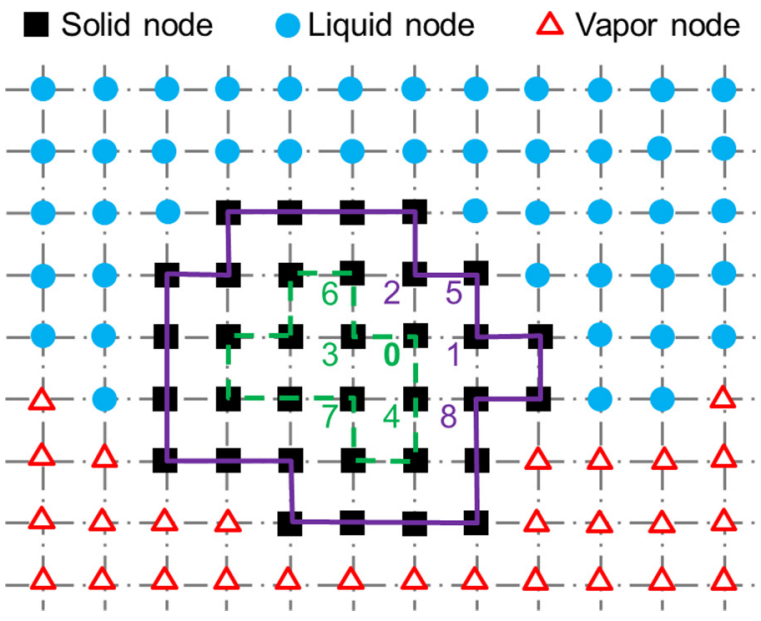

FIG. 3. Illustration of implementing no flux boundary condition at solid-liquid and solid-vapor interface. Solid purple line and dashed green line indicate first and second layer of solid object, respectively.

simulation is higher to obtain the same qualitative deposition results. The initial particle concentration is determined from the top view of the experimental final configuration, shown in Fig. 4(a). From the experiment result shown in Fig. 4 below, we can calculate the ratio $\left(A_{N, p}\right)$ of particle bridge area $\left(A_{p}\right)$ over solid pillar area $\left(A_{s}\right)$; i.e., $A_{N, p}=A_{p} / A_{s}=93.5 \%$. Then, according to Fig. 2, the initial particle concentration is calculated as $\phi_{0}=A_{N, p} 2 d^{2} /\left(L W-2 d^{2}\right) \approx 0.12$. During evaporation, the liquid area decreases while the particles accumulate around the liquid-vapor interface and finally deposit as the solid structures. Zero flux boundary condition $\mathbf{u}_{p, \mathbf{n}} \phi+$ $D_{p} \partial \phi / \partial \mathbf{n}=0$ is applied at the fluid-solid boundary to allow particles to deposit and ensure total particle concentration to be conserved. The particle velocity is zero at the solid point; therefore the no flux boundary condition degenerates to $\partial \phi / \partial \mathbf{n}=0$. Moreover, since the fluid-solid boundary can be very irregular during particle deposition, accurate boundary condition implementation can be very challenging.

Here we propose a unilateral interpolation method with the weight factors $W_{i}^{\prime}, i \in\{1, \ldots, 8\}$ imitated from the ones $\left(W_{i}=1 / 3, i \in\{1, \ldots, 4\}, W_{i}=1 / 12, i \in\{5, \ldots, 8\}\right)$ in isotropic central schemes in [66]. As illustrated in Fig. 3, the black squares, blue circles, and red triangles represent solid, liquid, and vapor nodes, respectively. At the first layer ( $\partial S_{1}$, solid purple line) of the solid body $(S)$, the modified convection diffusion equation [Eq. (11)] is still applied. To achieve $\partial \phi / \partial \mathbf{n}=0$ at $\partial S_{1}$, we modify the concentration at the second layer ( $\partial S_{2}$, dashed green line) of $S$. The unilateral interpolation is, for $\forall j \in \partial S_{2}$,

$$
\phi_{j}=\sum_{i=1}^{8} W_{i}^{\prime} \phi_{i}, \quad W_{i}^{\prime}=W_{i, m} / \sum_{i=1}^{8} W_{i, m}
$$

with the modified weight factors

$$
W_{i, m}=\left\{\begin{array}{l}
1 / 3, i \in\{1, \ldots, 4\}, i \in \partial S_{1} \\
1 / 12, \quad i \in\{5, \ldots, 8\}, i \in \partial S_{1} . \\
0, \quad \text { otherwise }
\end{array}\right.
$$

(a)

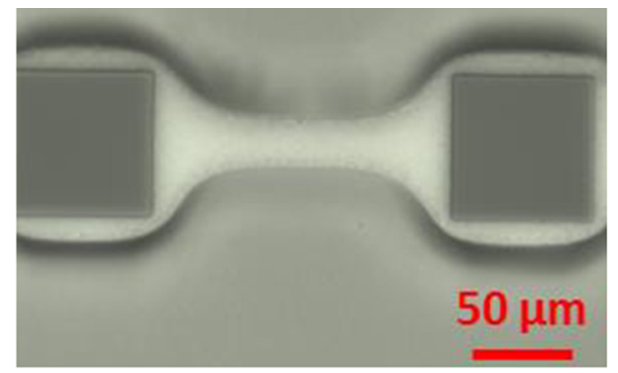

(b)

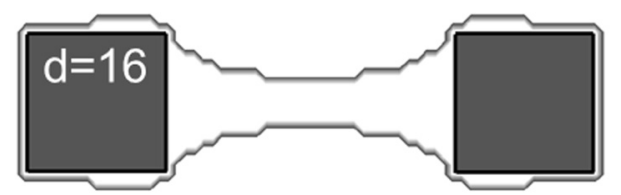

(c)

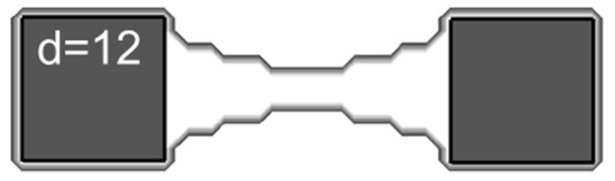

(d)

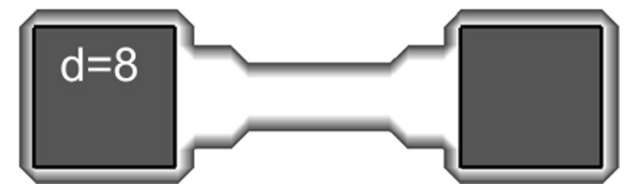

FIG. 4. Comparison of bridge profiles (light gray) between experiment (a) from Ref. [6] and simulations of pillars (dark gray) with size $16 \times 16(\mathrm{~b}), 12 \times 12$ (c), and $8 \times 8$ (d) lattices ${ }^{2}$, respectively.

For example, the concentration at node 0 is $\phi_{0}=$ $\frac{2}{5}\left(\phi_{1}+\phi_{2}\right)+\frac{1}{10}\left(\phi_{5}+\phi_{8}\right) \quad$ with $\quad \sum_{i=1}^{8} W_{i, m}=5 / 6$. This boundary condition modifies the concentration values at $\partial S_{2}$. In the simulations, the real values of concentration at $\partial S_{2}$ are saved before being modified, so as to obtain the correct statistics of the total concentration. With the proposed no flux boundary condition, the variation of total particle concentration can be limited to less than $2.5 \%$ in our simulations.

\section{Simulation results}

We compare the profiles of solid bridges for different pillar sizes at the moment when the drying process is completed. The comparison is shown in Fig. 4, from which we can see that all bridges show similar profiles that agree with the experiment [6] qualitatively. With increasing resolution the profile becomes better resolved and as a consequence matches more closely with that observed in experiment. With nine processors of Dual socket Intel Xeon E5-2670, the computational times for the simulations of three different resolutions are 52, 150, and $360 \mathrm{~s}$, respectively. Considering both the accuracy and computational cost, in the following, we use a pillar size of $12 \times 12$ lattices $^{2}$ for simulations with a much larger number of pillars in the spiral-shaped micropore structure (SMS).

\section{B. Spiral-shaped micropore structure}

\section{Experimental geometry and setup}

In this section, we study the nonisothermal drying process of a colloidal suspension in a spiral-shaped micropore structure (SMS) with 220 square pillars. In the experimental 
(a)

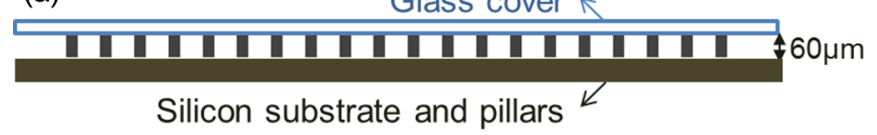

(b)

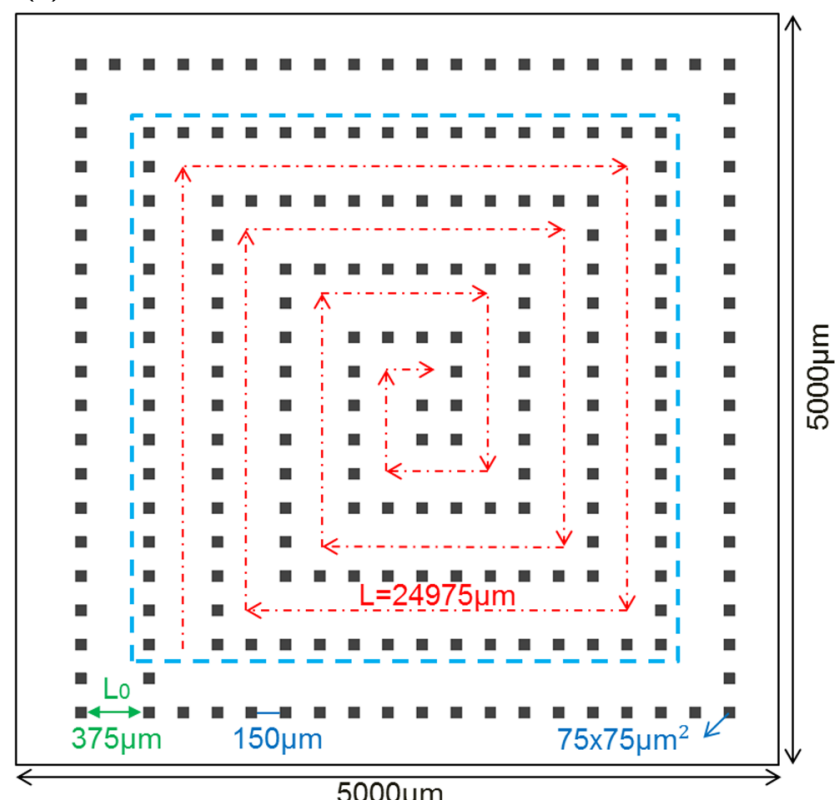

FIG. 5. Illustration of the spiral-shaped micropore structure (SMS) with dimensions: (a) side view; (b) top view. The red dasheddotted array denotes the receding path of the main liquid-vapor meniscus.

setup, the base substrate and the pillars of SMS are made of silicon while the top cover is made of glass for visualization purposes. The configurations and dimensions of SMS are illustrated in Fig. 5.

As described in detail in [6], the SMS is heated as it is deposited on an aluminum plate of temperature around 343.15 $\mathrm{K}\left(70^{\circ} \mathrm{C}\right)$. Then the colloidal suspension with $2.0 \%$ particle volume fraction is injected into it until saturation. The liquid is thus heated by the SMS. Due to the temperature difference between the colloidal suspension and the environment (40-45 $\mathrm{K}$ ), a nonisothermal evaporation is induced. Since the edges of the SMS are very rough due to fabrication, a portion of particles attaches to the edges; this deposition seriously affects the evaporation and particle deposition within the spiral at the beginning of the process, leading to noisy data at the periphery of the SMS. As shown in Fig. 6, we can neglect this initial period and the affected area by focusing on the main liquid-vapor meniscus once it has moved to the second loop of the spiral in the SMS. Thus, the experimental area that we consider in our simulation is the area delineated by blue dashed lines in Fig. 6, and we regard this state as the initial state $\left(t_{N}=0.00\right)$ for our analyses.

\section{Simulation geometry and setup}

Because of the high aspect ratio of the SMS (5000/60), the micropillar system is considered as a quasi-2D system and therefore we perform a 2D simulation. The geometry in our simulation is the same as seen in the top view of the SMS in

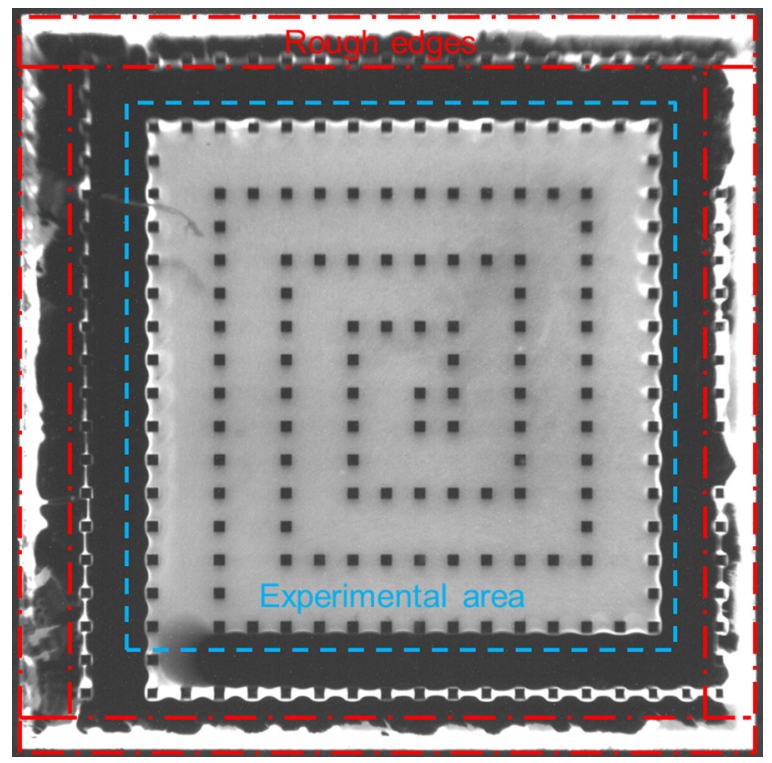

FIG. 6. Microscopic image of the experiment showing particle accumulation and deposition (bright white color) at the rough edges of SMS, while the solution is grayish. The experimental area that we considered is within the dashed blue line. This state is considered the initial state in the simulation.

Fig. 5. The simulation setup and boundary conditions are the same as in the two-micropillar system. With 225 processors of a dual socket Intel Xeon E5-2670, the computational time for the simulation is around $15 \mathrm{~h}$ and $16 \mathrm{~min}$. The initial particle concentration in our simulation is $9.0 \%$. We mention that the initial concentration we use is higher than that in the experiment $(2.0 \%)$ due to the difference between $2 \mathrm{D}$ and $3 \mathrm{D}$ bridge structures. In the experiment, the initial $2.0 \%$ particle concentration ends up around $17.1 \%$ of the deposited bridge area (not including the particles at the rough edges of the SMS), indicating that most of the depositions are somewhat hollow structures. However, in the 2D simulation, the deposition can be interpreted as a perfectly extruded geometry in the vertical direction, which consumes many more particles. Since the formed solid structures in the experiment do not bridge completely the interpillar space, being hollow, we assume that the particle depositions in the SMS are formed of two equal, separated layers attached to the glass cover and silicon substrate together with a blocking ratio of 2 [69]. Then a corresponding initial concentration for $2 \mathrm{D}$ simulation to get the same deposition should be $17.1 \% / 2=8.55 \%$, which is very close to $9.0 \%$ in our simulation.

To analyze effectively the dynamic process of drying of the colloidal suspension, a nondimensional time is defined as $t_{N}=t /\left(L_{0} / \bar{U}\right)$, where $L_{0}$ is the characteristic length taken as the distance between two square pillars (see Fig. 5). $\bar{U}$ is the average velocity of the liquid front calculated as $\bar{U}=L / t_{e}$, where $L$ is defined as the dimension of the porous medium as shown in Fig. 5 and $t_{e}$ is the total evaporation time of the colloidal suspension in the area delimited in Fig. 6.

\section{Qualitative analyses}

The mechanisms of pure liquid drying have been discussed for similar SMSs in [67] in detail. The nondimensional 
(a) Experiment

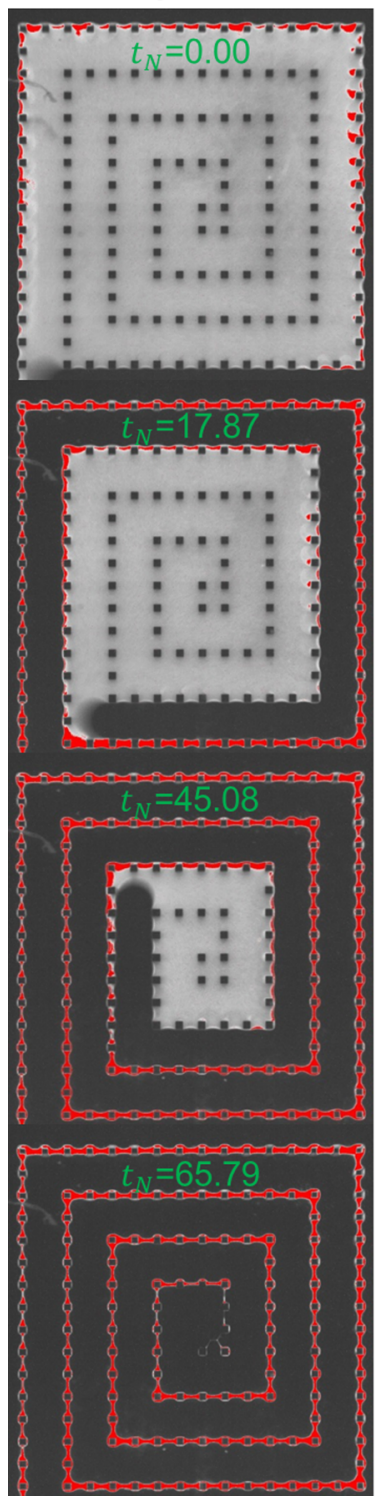

(b) Hybrid LBM

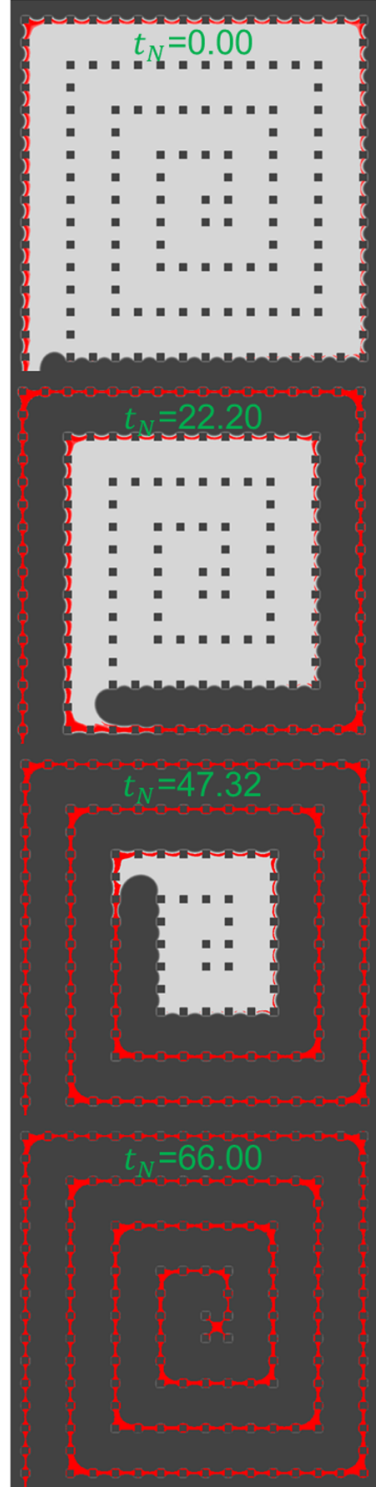

FIG. 7. Comparison of liquid configuration (light gray color) and particle accumulation and deposition (dark red color) at different nondimensional times during drying of colloidal suspensions in SMS. Left: experiment. Right: simulation.

numbers that describe the mechanisms are Reynolds number for liquid $\left(\operatorname{Re}_{L}\right)$ and air $\left(\operatorname{Re}_{V}\right)$, and capillary number $(\mathrm{Ca})$ and Péclet number for vapor transport $\left(\mathrm{Pe}_{V}\right)$. With the parameters chosen in our simulations, the corresponding nondimensional numbers are $\operatorname{Re}_{L}=3.20, \mathrm{Re}_{V}=1.15, \mathrm{Ca}=4.67 \times 10^{-2}$, $\mathrm{Pe}_{V}=2.10 \times 10^{-2}$, and $\mathrm{Pe}_{p}=1.53 \times 10^{2}$, and they reflect that the liquid and vapor flows are laminar flows, the capillary force is dominant, the vapor transport is diffusive, and the particle transport in the liquid phase is convective. Figure 7 compares the liquid configuration and particle accumulation and deposition during drying of colloidal suspensions in SMS (see the Supplemental Material [70] for the videos of the drying process of simulation and experiment). The light gray area indicates the suspension while the areas highlighted in red represent the accumulation and deposition of particles. In

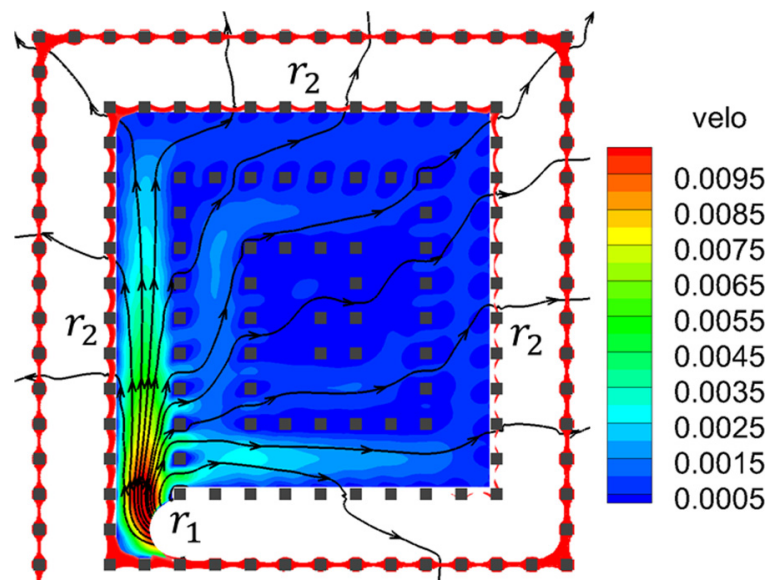

FIG. 8. Internal flow of colloidal liquid (capillary pumping) from large meniscus to small ones at nondimensional time $t_{N}=22.20$, driven by capillary pressure difference. The streamlines outside the colloid suspension indicate evaporated vapor flow. The contour represents the velocity magnitude of internal flow.

the experimental images on the left side, we can see that, at nondimensional time $t_{N}=0.00$, some particles have already accumulated along the liquid-vapor interface. Since there is still colloidal liquid, no solid particle bridge is formed yet. At $t_{N}=17.87$, the drying colloidal suspension has receded to an inner loop, and accumulated particles first seen at $t_{N}=0.00$ now form deposited solid bridges between pillars at the first row of pillars, while further accumulation is ongoing along the second inner row. As drying continues, the particles keep accumulating and depositing layer by layer from outside to inside, until the colloidal suspension is fully dried up. Finally, at $t_{N}=65.79$, the deposited particles generate a spiral-shaped solid bridge connecting all the pillars.

The selected snapshots on the right side of Fig. 7 show our hybrid LBM simulation results at nondimensional times similar to the experimental ones shown. It is seen that, for both colloidal liquid configuration and particle accumulation and deposition, results obtained by simulations are qualitatively in very good agreement with experimental ones. A small difference between simulation and experiment is seen at $t_{N}=$ 65.79 as the walls of the last five pillars are faint in the experiment. This can be explained by an extra depletion of the colloidal suspension in the experiment as some particles are lost at the rough edges of the SMS, as already shown in Fig. 6.

To further understand how the particles are transported and accumulate during drying, we analyze the flow inside the colloid suspension. In Fig. 8, we plot the velocity magnitude inside the colloid suspension representing the streamlines for flow of the colloidal suspension and the evaporated vapor. It is obvious that the colloidal suspension flows from the large meniscus $\left(r_{1}\right)$ to small ones $\left(r_{2}\right)$, driven by the difference of capillary force, i.e., capillary pumping $[58,71]$. The particles are passively transported by the colloidal liquid, arriving at the small menisci. Due to the fluid-particle force $\mathbf{F}_{p}$ proposed in Eq. (13), the particles cannot flow into the vapor phase. Therefore, they can only accumulate at the liquidvapor interfaces. After the local colloidal suspension is totally 


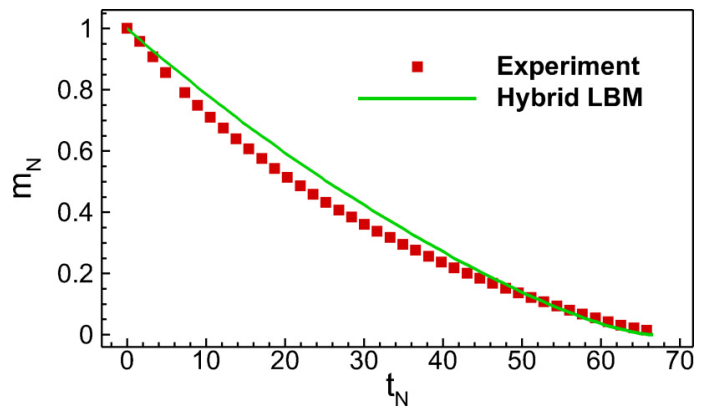

FIG. 9. Comparison of normalized colloidal liquid mass between experiment (red solid square) and hybrid LBM simulation (green solid line) during drying of colloidal suspensions in SMS.

evaporated, the accumulated particles finally deposit as solid bridges between pillars as shown in Fig. 8. The streamlines outside of colloid suspension represent the flow direction of the evaporated vapor. As explained in Sec. II C, the bridges do not clog the vapor path since they are quite hollow structures that vapor can actually penetrate in the experiment. As seen in Fig. 7, the suspension of $2.0 \%$ particle concentration ends up to around $17.1 \%$ of the deposition area; thus only around $(2.0 / 17.1) \times 100 \%=11.7 \%$ of deposited vertical bridges are actually occupied with particles. This is the reason we allow that the evaporated vapor can be transported through the bridge structures in our simulation by applying one-way coupling of particle transport and deposition to the nonisothermal drying model.

\section{Quantitative analyses}

To further verify quantitatively the accuracy of our model, we investigate the variables that represent the process of colloidal suspension drying and particle deposition. We define the normalized liquid mass $m_{N}$, evaporation rate $E p_{N}$, and particle deposition area $A_{N, p}$ as

$$
\begin{aligned}
m_{N}\left(t_{N}\right) & =m\left(t_{N}\right) / m\left(t_{N}=0\right) \\
E p_{N}\left(t_{N}\right) & =\left[m_{N}\left(t_{N+1}\right)-m_{N}\left(t_{N}\right)\right] /\left(t_{N+1}-t_{N}\right), \\
A_{N, p}\left(t_{N}\right) & =A_{p}\left(t_{N}\right) / A_{s},
\end{aligned}
$$

where $m\left(t_{N}=0\right)$ and $A_{s}$ are the initial liquid mass and solid pillar area in the experiment area of Fig. 6, respectively.

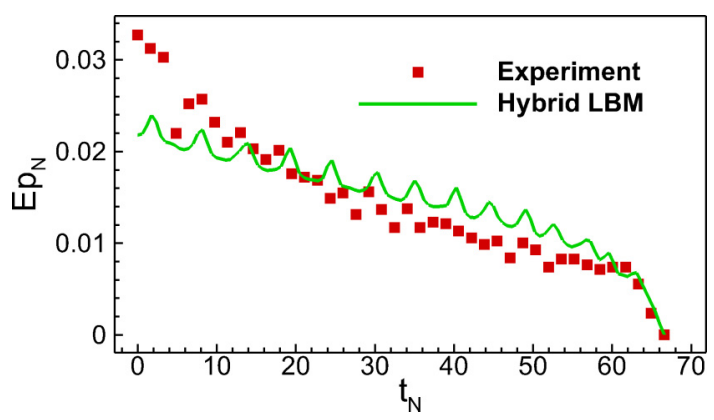

FIG. 10. Comparison of colloidal liquid evaporation rate between experiment (red solid square) and hybrid LBM simulation (green solid line) during drying of colloidal suspensions in SMS.

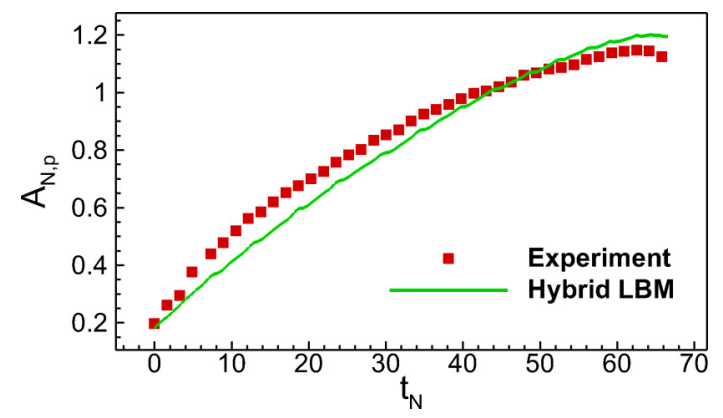

FIG. 11. Comparison of colloidal particle deposition area between experiment (red solid square) and hybrid LBM simulation (green solid line) during drying of colloidal suspensions in SMS.

Simulation results can be converted to physical units using the conversion method explained in Appendix D in [67].

The time evolutions of normalized liquid mass $m_{N}$ from both experiment and hybrid LBM simulation are compared in Fig. 9. The simulation results are in a close match with experimental observations. For the evaporation rate $E p_{N}$ shown in Fig. 10, the rate decreases slowly with time for both experiment and simulation, as a result of reduced liquidvapor interface [67]. When the main meniscus passes the corner of the SMS, a small isolated colloidal liquid cluster is formed, resulting in a slight increase of the evaporation rate at each corner [67]. This effect is shown as small peaks in the evaporation rate in the simulation results in Fig. 10. In the experiment, the drying process is $3 \mathrm{D}$ and more continuous, but the perturbations can still be seen. For the accumulation and deposition of colloidal particles, the variation of deposited area $A_{N, p}$ as a function of time is shown in Fig. 11. The general profiles for both experiment and simulation are convex and very similar. The convex shape means that the deposited area $A_{N, p}$ increases slower and slower with drying, which can be explained by the decrease of evaporation rate. For the final deposition area, there is a $6.25 \%$ difference between the hybrid LBM simulation and the experiment. This discrepancy may be due to the three reasons mentioned above, namely: first, the initial concentration in simulation and experiment are different (9.0\% for simulation and $2.0 \%$ for experiment); second, the experiment is $3 \mathrm{D}$ and the deposited area is not identical along the vertical direction; third, some particles are lost near the rough edges outside of the experimental area as shown in Fig. 6. We expect that we can reduce this difference with $3 \mathrm{D}$ simulations in future work. From these quantitative comparisons of variables like $m_{N}, E p_{N}$, and $A_{N, p}$ between experiments and simulations, we conclude that the results obtained by the tricoupled LBM model are sufficiently accurate to capture the experimental observations of nonisothermal drying of the colloidal suspension.

\section{CONCLUSION}

In this paper, we proposed a tricoupled hybrid LBM which is capable of modeling the nonisothermal drying of colloidal suspensions and the deposition of the colloidal particles. The hybrid model consists of three parts: an entropic multiplerelaxation-time multirange pseudopotential LBM (EMRT-MP LBM) for isothermal two-phase flow, an extended temperature 
equation (ETE) for heat transport and transfer (latent heat), and a modified convection diffusion equation (MCDE) for particle transport and deposition. T-EMRT-MP LBM is a two-way coupling of EMRT-MP LBM and ETE for nonisothermal liquid drying, and MCDE is further coupled (one way) with T-EMRT-MP LBM for nonisothermal drying of the colloidal suspension. The drying of the colloidal suspension in a two-pillar system is simulated as a simple validation case, with three different resolutions. The deposited profiles of particles are very similar and they approach experimental results by increasing the resolution. The nonisothermal drying of a colloidal suspension within a 220-pillar complex system (SMS) is simulated and the results are compared with the experimental results. Qualitatively, both the simulated colloidal suspension configuration and colloidal particle accumulation and deposition agree well with the experimental ones, during the entire drying process. Quantitatively, the evolutions of simulated variables including liquid mass, evaporation rate, and particle deposition area show good agreement with the experimental ones. All the comparisons between simulation and experimental results indicate that our proposed hybrid LBM is accurate in modeling nonisothermal drying of colloidal suspensions in a complex system.

\section{ACKNOWLEDGMENTS}

Swiss National Science Foundation (SNF, Project No. 160189) is acknowledged for the financial support. LANL's Institutional Computing Program and the Swiss National Super Computing Center (Project No. s823) are acknowledged for providing the computing support.
[1] R. D. Deegan, O. Bakajin, T. F. Dupont, G. Huber, S. R. Nagel, and T. A. Witten, Nature 389, 827 (1997).

[2] P. J. Yunker, T. Still, M. A. Lohr, and A. G. Yodh, Nature 476, 308 (2011).

[3] J. Park and J. Moon, Langmuir 22, 3506 (2006).

[4] T. Brunschwiler, J. Zürcher, L. Del Carro, G. Schlottig, B. Burg, S. Zimmermann, U. Zschenderlein, B. Wunderle, F. SchindlerSaefkow, and R. Stässle, J. Electron. Packag. 138, 041009 (2016).

[5] J. Zurcher, X. Chen, B. R. Burg, S. Zimmermann, R. Straessle, A. R. Studart, and T. Brunschwiler, IEEE Trans. Compon., Packag., Manuf. Technol. 6, 1785 (2016).

[6] R. Stadler, L. D. Carro, J. Zurcher, G. Schlottig, A. R. Studart, and T. Brunschwiler, in Proceedings of 16th IEEE Intersociety Conference on Thermal and Thermomechanical Phenomena in Electronic Systems (ITherm) (IEEE, Piscataway, NJ, 2017), p. 167.

[7] C. Hamon, M. Postic, E. Mazari, T. Bizien, C. Dupuis, P. EvenHernandez, A. Jimenez, L. Courbin, C. Gosse, F. Artzner, and V. Marchi-Artzner, ACS Nano 6, 4137 (2012).

[8] M. A. Boles, M. Engel, and D. V. Talapin, Chem. Rev. 116, 11220 (2016).

[9] U. Frisch, B. Hasslacher, and Y. Pomeau, Phys. Rev. Lett. 56, 1505 (1986).

[10] F. Bösch, S. S. Chikatamarla, and I. V. Karlin, Phys. Rev. E 92, 043309 (2015).

[11] L. Jahanshaloo, E. Pouryazdanpanah, and N. A. Che Sidik, Numer. Heat Transfer, Part A 64, 938 (2013).

[12] F. Qin, A. Mazloomi Moqaddam, Q. Kang, D. Derome, and J. Carmeliet, Phys. Fluids 30, 032104 (2018).

[13] A. Mazloomi M, S. S. Chikatamarla, and I. V. Karlin, Phys. Rev. Lett. 114, 174502 (2015).

[14] S. Son, L. Chen, D. Derome, and J. Carmeliet, Comput. Fluids 117, 42 (2015).

[15] A. M. Moqaddam, D. Derome, and J. Carmeliet, Langmuir 34, 5635 (2018).

[16] M. Wöhrwag, C. Semprebon, A. Mazloomi Moqaddam, I. Karlin, and H. Kusumaatmaja, Phys. Rev. Lett. 120, 234501 (2018).

[17] X. Shan, Phys. Rev. E 55, 2780 (1997).
[18] I. V. Karlin, D. Sichau, and S. S. Chikatamarla, Phys. Rev. E 88, 063310 (2013).

[19] A. J. C. Ladd and R. Verberg, J. Stat. Phys. 104, 1191 (2001).

[20] U. Lantermann and D. Hanel, Comput. Fluids 36, 407 (2007).

[21] X. Shan and H. Chen, Phys. Rev. E 49, 2941 (1994).

[22] X. Shan, Phys. Rev. E 77, 066702 (2008).

[23] A. Mazloomi M., S. S. Chikatamarla, and I. V. Karlin, Phys. Rev. E 92, 023308 (2015).

[24] F. Bösch, B. Dorschner, and I. Karlin, EPL 122, 14002 (2018).

[25] A. K. Gunstensen, D. H. Rothman, S. S. Zaleski, and G. Zanetti, Phys. Rev. A 43, 4320 (1991).

[26] A. Fakhari and M. H. Rahimian, Phys. Rev. E 81, 036707 (2010).

[27] H. Liu, A. J. Valocchi, Y. Zhang, and Q. Kang, Phys. Rev. E 87, 013010 (2013).

[28] N. Frapolli, S. S. Chikatamarla, and I. V. Karlin, Phys. Rev. E 90, 043306 (2014).

[29] N. Frapolli, S. S. Chikatamarla, and I. Karlin, J. Stat. Phys. 161, 1434 (2015).

[30] G. Gonnella, A. Lamura, and V. Sofonea, Phys. Rev. E 76, 036703 (2007).

[31] Y. Gan, A. Xu, G. Zhang, and S. Succi, Soft Matter 11, 5336 (2015).

[32] N. I. Prasianakis and I. V Karlin, Phys. Rev. E 78, 016704 (2008).

[33] N. I. Prasianakis and I. V Karlin, Phys. Rev. E 76, 016702 (2007).

[34] N. I. Prasianakis, S. S. Chikatamarla, I. V Karlin, S. Ansumali, and K. Boulouchos, Math. Comput. Simul. 72, 179 (2006).

[35] R. Zhang and H. Chen, Phys. Rev. E 67, 066711 (2003).

[36] S. Gong and P. Cheng, Int. J. Heat Mass Transfer 55, 4923 (2012).

[37] Q. Li, P. Zhou, and H. J. Yan, Phys. Rev. E 96, 063303 (2017).

[38] Q. Li, Q. J. Kang, M. M. Francois, Y. L. He, and K. H. Luo, Int. J. Heat Mass Transfer 85, 787 (2015).

[39] Q. Li, P. Zhou, and H. J. Yan, Langmuir 32, 9389 (2016).

[40] Z. Chai and T. S. Zhao, Phys. Rev. E 87, 063309 (2013).

[41] Q. Li and K. H. Luo, Phys. Rev. E 89, 053022 (2014).

[42] Y. Mu, L. Chen, Y. He, Q. Kang, and W. Tao, Int. J. Heat Mass Transfer 106, 708 (2017). 
[43] S. A. Hosseini, N. Darabiha, D. Thévenin, and A. Eshghinejadfard, Int. J. Mod. Phys. C 28, 1750141 (2017).

[44] J. Perko and R. A. Patel, Phys. Rev. E 89, 053309 (2014).

[45] R. Verberg, J. M. Yeomans, and A. C. Balazs, J. Chem. Phys. 123, 224706 (2005).

[46] X. Shi, Ph.D. thesis, University of Delaware, 2016.

[47] L. K. Limbach, Y. Li, R. N. Grass, T. J. Brunner, M. A. Hintermann, M. Muller, D. Gunther, and W. J. Stark, Environ. Sci. Technol. 39, 9370 (2005).

[48] S. A. Bradford and N. Toride, J. Environ. Qual. 36, 1346 (2007).

[49] N. Islam, S. J. Miklavcic, B. H. Bradshaw-Hajek, and L. R. White, PLoS ONE 12, e0183127 (2017).

[50] Y. Chen, Q. Kang, Q. Cai, M. Wang, and D. Zhang, Commun. Comput. Phys. 18, 757 (2015).

[51] A. S. Joshi and Y. Sun, Phys. Rev. E 82, 041401 (2010).

[52] J. B. Miller, A. C. P. Usselman, R. J. Anthony, U. R. Kortshagen, A. J. Wagner, A. R. Denton, and E. K. Hobbie, Soft Matter 10, 1665 (2014).

[53] K. Yang and Z. Guo, Comput. Fluids 124, 157 (2016).

[54] C. Ancey, P. Bohorquez, and J. Heyman, J. Geophys. Res.: Earth Surf. 120, 2529 (2015).

[55] Q. Kang, D. Zhang, P. C. Lichtner, and I. N. Tsimpanogiannis, Geophys. Res. Lett. 31, 1 (2004).

[56] A. Cartalade, A. Younsi, and M. Plapp, Comput. Math. Appl. 71, 1784 (2016).

[57] M. N. Rad and N. Shokri, Geophys. Res. Lett. 39, L04403 (2012).
[58] F. Qin, A. Mazloomi Moqaddam, Q. Kang, D. Derome, and J. Carmeliet, Transp. Porous Media (2018), doi: 10.1007/s11242-018-1157-4.

[59] I. V. Karlin, F. Bösch, and S. S. Chikatamarla, Phys. Rev. E 90, 031302(R) (2014).

[60] I. V Karlin, A. Ferrante, and H. C. Öttinger, Europhys. Lett. 47, 182 (1999).

[61] S. S. Chikatamarla, S. Ansumali, and I. V. Karlin, Phys. Rev. Lett. 97, 010201 (2006).

[62] M. Sbragaglia, R. Benzi, L. Biferale, S. Succi, K. Sugiyama, and F. Toschi, Phys. Rev. E 75, 026702 (2007).

[63] P. Yuan and L. Schaefer, Phys. Fluids 18, 042101 (2006).

[64] D. M. Anderson, G. B. McFadden, and A. A. Wheeler, Annu. Rev. Fluid Mech. 30, 139 (1998).

[65] Y. Yu, Q. Li, C. Q. Zhou, P. Zhou, and H. J. Yan, Appl. Therm. Eng. 127, 1346 (2017).

[66] T. Lee and C. L. Lin, J. Comput. Phys. 206, 16 (2005).

[67] F. Qin, L. Del Carro, A. M. Moqaddam, Q. Kang, T. Brunschwiler, D. Derome, and J. Carmeliet, J. Fluid Mech. 866, 33 (2019).

[68] Q. Kang, P. C. Lichtner, and D. Zhang, J. Geophys. Res. Solid Earth 111, B05203 (2006).

[69] M. Su, Z. Huang, Y. Li, X. Qian, Z. Li, X. Hu, Q. Pan, F. Li, L. Li, and Y. Song, Adv. Mater. 30, 1703963 (2018).

[70] See Supplemental Material at http://link.aps.org/supplemental/ 10.1103/PhysRevE.99.053306 for the videos of the drying process of simulation and experiment.

[71] A. G. Yiotis, A. K. Stubos, A. G. Boudouvis, and Y. C. Yortsos, Adv. Water Resour. 24, 439 (2001). 